\title{
Incidentalomas adrenales. Unas sorpresas inesperadas
}

\section{Arturo Orrego M.}

Médico Internista Endocrinólogo, Miembro Honorario de la Asociación Colombiana de Endocrinología, Diabetes y Metabolismo

Fecha de recepción: 2/02/2017

Fecha de aceptación: 13/02/2017

$\mathrm{E}$ 1941 se informó por primera vez la existencia de un Incidentaloma adrenal, desde entonces, gracias a los avances en las técnicas imaginológicas en el diagnóstico de este hallazgo ha sido, cada vez más prevalente ${ }^{(1,2)}$. Lo más importante en la identificación de estas lesiones es definir si son potencialmente malignas o pueden llegar a serlo. En esta revisión se expondrá un acercamiento práctico en la investigación y el seguimiento de los incidentalomas adrenales de acuerdo con la experiencia de varios autores que se han ocupado de este tema ${ }^{(1-16)}$.

\section{Definición}

Los incidentalomas adrenales son masas de estas glándulas descubiertas al azar durante una evaluación radiológica no dirigida a detectarlos, en la ausencia de manifestaciones clínicas sugestivas de enfermedades adrenales. La definición generalmente comprende lesiones mayores de $1 \mathrm{~cm}$ que son detectadas por fuera del estado de invasión de cánceres bien identificados. Si por error o descuido no se hizo el diagnóstico de enfermedad adrenal durante la evaluación clínica, no se pueden clasificar, en el estricto sentido de la palabra, como un incidentaloma adrenal.

\section{Estudios epidemiológicos}

En estudios de autopsia, la prevalencia de los incidentalomas adrenales ha sido de 1,1\% a 32\%(3-5). En 25 estudios de autopsia se encontró una prevalencia del $6 \%{ }^{(6)}$. Los incidentalomas adrenales son más frecuentes en pacientes con mayor edad. En un estudio se encontró que el 7\% de los pacientes autopsiados después de los 70 años presentaban un incidentaloma adrenal, en cambio solo el 1\% de los individuos autopsiados antes de los 30 años eran portadores de esta entidad ${ }^{(6)}$. Otros investigadores confirmaron la dependencia de la edad en la presentación de los incidentalomas adrenales ${ }^{(2)}$. No se ha observado diferencia de género en las autopsias.

\section{Estudios tomográficos computarizados}

Con estudios tomográficos tempranos en los años 80 se informó una prevalencia de los incidentalomas adrenales del
$0,9 \%{ }^{(2)}$. Con los nuevos aparatos de tomografía axial computarizada (TAC) se identificaron incidentalomas adrenales en el 4,4\%, en Italia, en el $2006^{(7)}$. En 2011, se encontró únicamente una prevalencia de $0,98 \%$ con TAC abdominal y de $0,81 \%$ con la TAC torácica ${ }^{(8)}$. Algunos estudios demostraron mayor prevalencia del incidentaloma en mujeres; en cambio los estudios de autopista han identificado una prevalencia igual en hombres y mujeres ${ }^{(9)}$.

\section{Causas de incidentalomas adrenales}

Se han identificado al menos 38 diagnósticos diferentes en los pacientes con incidentalomas adrenales ${ }^{(2)}$. Los más frecuentes son los adenomas corticales no funcionales (70 a 80\%), feocromocitomas $(1,1 \%$ a $11 \%)$, síndrome de Cushing subclínico ( $5 \%$ a $20 \%$ ), aldosteronismo primario (1 a $2 \%$ ) y metástasis $2,5 \%(2,4,10-14)$. En la tabla 1 se mencionan diferentes estados que pueden confundirse con incidentalomas adrenales ${ }^{(17)}$.

Tabla 1. Lesiones adrenales que se presentan como incidentalomas

1. Masas corticoadrenales

- Benignas: adenoma, hiperplasia nodular, hiperplasia adrenal congénita.

- Malignas: cáncer corticoadrenal primario

2. Tumores meduloadrenales: feocromocitoma, ganglioneuroma, neuroblastoma

3. Otros tumores adrenales:

- Benignos: mielolipoma, teratoma, hamartoma, lipoma, hemangioma linfagioma, tumor adrenal adenomatoide.

- Malignos: metastásico, linfoma adrenal primario, melanoma adrenal primario.

4. Infecciones: hongos (histoplasmosis, coccidioidomicosis, blastomicosis), viral (citomegalovirus), parásitos (equinococosis), bacterial (tuberculosis, sífilis).

5. Infiltración: sarcoidosis, amiloidosis.

6. Quistes y seudoquistes.

7. Hemorragia, hematoma.

8. No trastornos adrenales: schwanoma, leiomiosarcoma, lipoma retroperitoneal.

- Se pueden presentar como masas bilaterales. 


\section{Examen clínico}

Una evaluación clínica exhaustiva puede ser de gran importancia porque la presencia de un incidentaloma adrenal puede producir secreciones hormonales que podrían dar al traste con la vida si no se detectan y tratan tempranamente. Debe investigarse el exceso de cortisol, de aldosterona, de catecolaminas, de andrógenos y de trastornos gastrointestinales. La presencia de hipertensión arterial reciente, de trastornos del metabolismo de la glucosa y pérdida de hueso pueden sugerir hipersecreción de cortisol. La aparición de crisis paroxística, de hipertensión arterial, las fluctuaciones de la presión arterial durante la anestesia, los eventos sincopales y los cambios posturales de la hipertensión arterial pueden sugerir la presencia de un feocromocitoma.

Los cánceres de pulmón, mama, renales y el melanoma tienen tendencia a hacer metástasis en las glándulas adrenales. Debe investigarse la presencia de malignidad y pérdida de peso. La combinación del exceso de cortisol y de andrógenos en presencia de una masa adrenal podría alertar a la probable presencia de un carcinoma adrenal primario.

Debe investigarse sobre la presencia familiar de la neoplasia endocrina múltiple tipo 2, el síndrome de von HippelLindau, la neurofibromatosis tipo 1 y el complejo de Carney. Se pueden observar masas bilaterales en pacientes con hiperplasia adrenal congénita, ya sean homocigotos o heterocigotos ${ }^{(18)}$. Sin embargo, tal asociación puede originarse en el aumento de las glándulas adrenales o en trastornos de actividad enzimática intratumoral, más que en un defecto enzimático verdadero $^{(19)}$.

\section{Estudios radiológicos}

Con la detección de un incidentaloma adrenal se debe determinar si la masa encontrada necesita someterse a un extenso protocolo de investigación. La TAC con medio de contraste puede no dar información suficiente sobre el riesgo de malignidad de la masa adrenal en estudio y puede necesitarse la realización de una TAC sin medio de contraste. Además, los incidentalomas adrenales detectados por ultrasonido o medicina nuclear, necesitan investigaciones diagnósticas posteriores.

\section{Selección de los estudios imaginológicos adrenales}

La TAC es el método más útil e importante en el estudio de los incidentalomas adrenales para definir el tamaño, el contenido lipídico y las características tumorales, la heterogeneidad, las calcificaciones, los bordes irregulares, la invasión local y las áreas de necrosis. Todas estas características radiológicas pueden ayudar a definir si existe probable malignidad. La resonancia magnética puede definir si existe probable malignidad, pero es más costosa y se prefiere dejarla para casos en los cuales la TAC está contraindicada o no sería capaz de definir los riesgos de malignidad. Los estudios imaginológicos funcionales de la glándula adrenal tienen un papel limitado en el estudio de casos seleccionados de pacientes con incidentaloma adrenal.

\section{Tamaño de los incidentalomas adrenales}

Tradicionalmente, el riesgo de malignidad de un incidentaloma adrenal se ha supuesto que está en relación con su tamaño. Musella y colaboradores ${ }^{(20)}$ encontraron durante la cirugía, que los incidentalomas adrenales carcinomatosos median más de $6 \mathrm{~cm}$, en cambio solamente el 1,2\% eran cancerosos con diámetros entre los 4 y $6 \mathrm{~cm}$ y no encontraron cáncer en incidentalomas menores de $4 \mathrm{~cm}$. Otros investigadores ${ }^{(21)}$ encontraron incidentalomas adrenales malignos en el $10 \%$ de lesiones con diámetros menores a $4 \mathrm{~cm}, 20 \%$ en tumores mayores de $6 \mathrm{~cm}$ y $47 \%$ en incidentalomas adrenales mayores de $8 \mathrm{~cm}$. Sin embargo, no debe olvidarse que existen informes de la presencia de incidentalomas adrenales malignos en tumores menores de $4 \mathrm{~cm}$.

El tamaño tumoral no es el único requisito para definir si un incidentaloma adrenal es maligno, sino que deben tenerse en cuenta otros hallazgos radiológicos, la presencia de áreas de necrosis, calcificaciones o invasión local y el coeficiente tomográfico de atenuación (menos de 10 unidades Hounsfield), con la TAC, sin medio de contraste $\mathrm{e}^{(23,24,25)}$.

\section{Imágenes con TAC}

Este método radiológico debe ser el primero utilizado en la evaluación de las masas adrenales. Da información sobre el tamaño del tumor adrenal, su densidad, las calcificaciones, áreas de necrosis presentes y la invasión local (tabla 2).

\section{Detección con TAC del coeficiente de atenuación}

Un valor bajo de atenuación de un nódulo adrenal es secundario a su alto contenido de grasa, de los esteroides producidos por el tejido cortical adrenal y tiene una especificidad cercana al $100 \%$ en el diagnóstico de adenoma, útil en la diferenciación de las lesiones benignas de las malignas ${ }^{(18,23)}$. La combinación de una masa adrenal de menos de $4 \mathrm{~cm}$ y un valor de atenuación menor o igual a 20 unidades Hounsfield (HU), tiene una especificidad del $100 \%$ para reconocer una lesión benigna. El valor de atenuación obtenido con TAC sin medio de contraste es mejor predictor de lesiones benignas que el tamaño de las lesiones adrenales ${ }^{(23,24)}$.

Los carcinomas adrenales y el feocromocitoma tienen un valor de atenuación mayor de $30 \mathrm{HU}$. El valor más bajo de atenuación del feocromocitoma ha sido de $17 \mathrm{HU}^{(25)}$. 
Tabla 2. Características de las imágenes gamagráficas más comunes en los incidentalomas adrenales

La necrosis, las hemorragias, las calcificaciones, los bordes irregulares y la invasión local son más frecuentes en los pacientes con carcinoma adrenal y feocromocitoma.

\begin{tabular}{|c|c|c|c|c|}
\hline Tipo & Tamaño (cm) & $\begin{array}{l}\text { Atenuación sin medio } \\
\text { de contraste }\end{array}$ & Lavado $>60 \%$ (absoluto) & $\begin{array}{l}\text { Pérdida en la intensidad } \\
\text { de señales en T1 (RM) }\end{array}$ \\
\hline Adenoma cortical & Pequeño $(<4)$ & Baja 70\% (<10 HU) & Sí & Sí \\
\hline Carcinoma cortical & Grande $(>4)$ & Alta $(>30 \mathrm{HU})$ & No & No \\
\hline Metástasis & Variable & $\operatorname{Alta}(>10 \mathrm{HU})$ & No, excepto en RCC y HCC & No, excepto en RCC y HCC \\
\hline Feocromocitoma & Grande (>3) & Alta $(>17 \mathrm{HU})$ & Variable & No \\
\hline
\end{tabular}

HCC: carcinoma hepatocelular; RCC, carcinoma renal; RM, resonancia magnética

\section{La TAC, el lavado y los patrones de reforzamiento}

Un valor del coeficiente de atenuación con TAC, sin medio de contraste de $\leq 10 \mathrm{HU}$, es un excelente predictor de benignidad. El 20\% a 30\% de los adenomas adrenales son pobres en lípidos $^{(26)}$. Lo que significa que no existen suficientes esteroles para disminuir el coeficiente de atenuación total del nódulo adrenal. En tales casos se necesitan imágenes adicionales para poder realizar el diagnóstico de adenoma y así tratar de evitar una cirugía inútil. Si la TAC sin medio de contraste muestra un valor mayor de $10 \mathrm{HU}$, se deben obtener imágenes después de la utilización de medio de contraste, a los 1 y 15 minutos para calcular el porcentaje de lavado. Un porcentaje de lavado mayor de $60 \%$ a los 15 minutos sugiere el diagnóstico de adenoma $^{(27,28,29)}$.

Con la resonancia magnética en fase $\mathrm{T} 1$ se pueden identificar lesiones ricas en lípidos por una reducción en la intensidad de señales. La presencia de una intensidad alta de señales en T2 en la resonancia magnética es característica, pero no patognomónica del feocromocitoma. Sin embargo, en el 35\% de los casos está ausente.

\section{Imágenes funcionales}

Las imágenes funcionales tienen muy poco valor en el diagnóstico y seguimiento de los incidentalomas adrenales. Se han utilizado estudios gamagráficos, empleando metayodobencilguanidina ( $\left.{ }^{123} \mathrm{I}-\mathrm{MIGB}\right)$, estudios con tomografía por emisión de fotones (PET), utilizando ${ }^{18} \mathrm{~F}$-fluoro-2-deoxiD-glucosa $\left({ }^{18} \mathrm{~F}_{-} \mathrm{FDG}\right),{ }^{18} \mathrm{~F}-3$,4-dihidroxifenilalanina $\left({ }^{18} \mathrm{~F}-\mathrm{DOPA}\right)$ $\mathrm{y}{ }^{18} \mathrm{~F}$-fluorodopamina $\left({ }^{18} \mathrm{~F}-\mathrm{FDA}\right)$. Estos isótopos se usan especialmente en presencia de feocromocitomas multicéntricos o metastásicos y tienen mayor sensibilidad que la $\mathrm{MIBG}^{(30,31,32)}$. El isótopo ${ }^{13}$ I-6b-yodometil-19-norcolesterol (NP59), no se recomienda para la evaluación de rutina de los incidentalomas adrenales ${ }^{(32)}$. Para masas adrenales pobres en grasa se sugiere utilizar en la TAC un medio de contraste para calcular el lava- do. La resonancia magnética se debe utilizar cuando la TAC no es concluyente o no se pueden administrar, sin peligro, medios yodados. Las gamagrafías funcionales podrían considerarse en casos para detectar feocromocitomas extradrenales o lesiones metastásicas.

\section{Evaluación bioquímica y conductas de manejo}

El 20\% de los incidentalomas adrenales producen síndrome de Cushing subclínico; aproximadamente el $2 \%^{(2,12,14,19,32)}$ dan origen a un hiperaldosteronismo primario; la prevalencia del feocromocitoma en pacientes con incidentaloma adrenal oscila entre $1,1 \%$ y $11 \%{ }^{(4,11,12,32)}$. Menos del $5 \%$ de los incidentalomas adrenales son carcinomas $^{(19)}$. Hasta el 30\% de los incidentalomas adrenales producen exceso de cortisol, catecolaminas o aldosterona, dependiendo de los criterios usados para definir la excesiva secreción hormonal ${ }^{(32)}$. Los pacientes portadores de incidentalomas adrenales pobres en grasa deben investigarse para feocromocitoma; el hiperaldosteronismo primario debe buscarse en pacientes con hipertensión y/o hipocaliemia. Debe ordenarse la medición de testosterona y dehidroepiandrosterona sulfato en las mujeres con virilización, con síntomas recientes sugestivos de hiperandrogenismo o con masas adrenales sospechosas de carcinoma adrenal ${ }^{(32)}$. Infortunadamente, solo entre $50 \%$ y $82 \%$ de los pacientes diagnosticados con incidentalomas adrenales son evaluados en la práctica clínica desde el punto de vista endocrino ${ }^{(8,33,34)}$.

En la tabla 2 se enumeran las imágenes gamagráficas más típicas de la mayoría de los tipos más comunes de incidentalomas adrenales.

\section{Seguimiento de los pacientes con incidentalomas adrenales}

El manejo de los pacientes con incidentalomas adrenales se resume en la figura 1. 


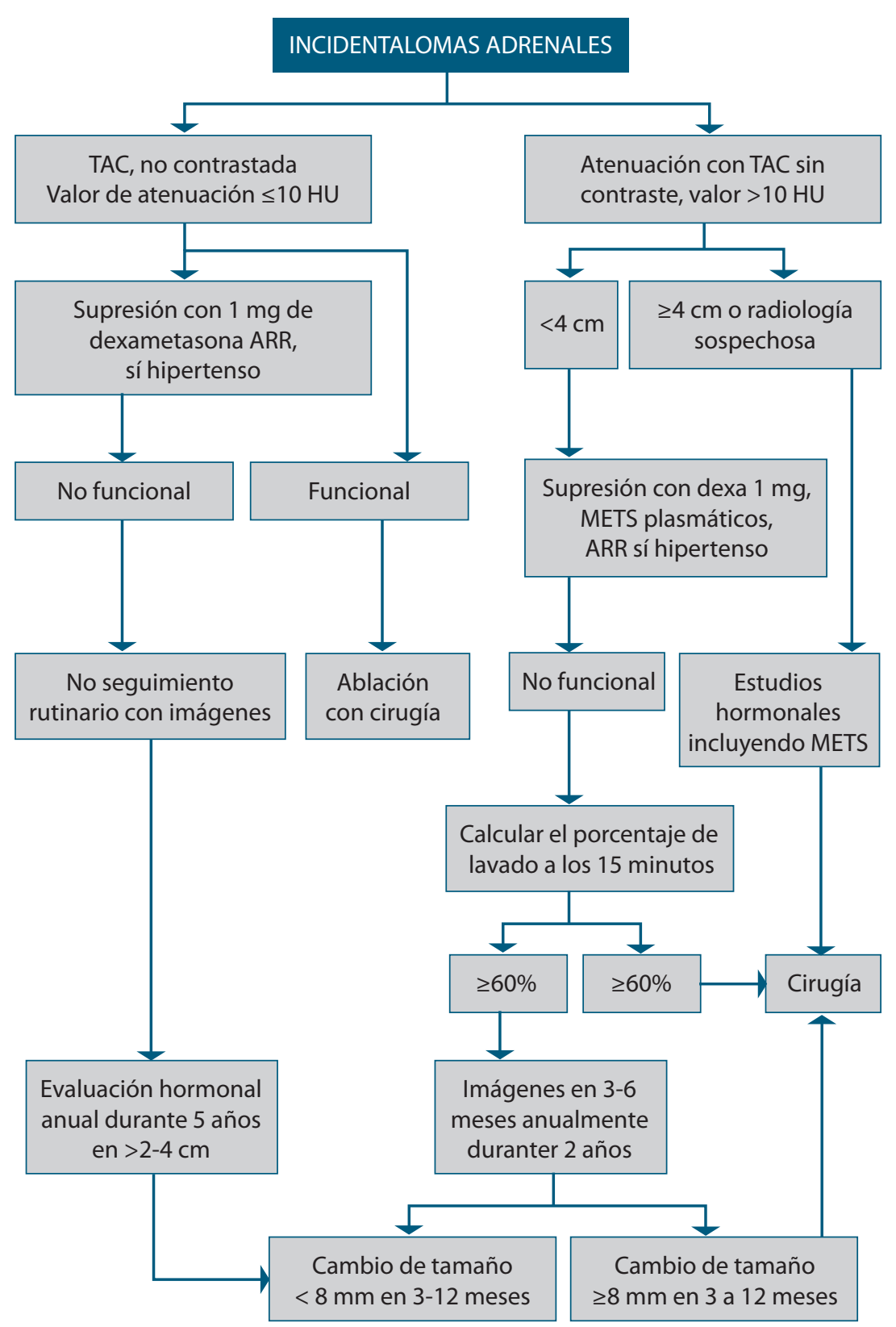

En un estudio prospectivo italiano ${ }^{(35)}$ encontraron que podría presentarse recurrencia del tumor después de los cinco años de seguimiento. Con base en lo anterior parece prudente, en los pacientes tratados de manera conservadora, hacer investigaciones bioquímicas y clínicas regularmente durante cinco años, hasta que nuevos estudios definan cuál es el seguimiento más apropiado y por cuánto tiempo.
Se sugiere repetir las imágenes adrenales anualmente durante 2 años. No existe evidencia suficiente que aconseje los estudios radiológicos muy frecuentes por el daño que la radiación puede producir en el paciente.

\section{Resumen}

Es de esperar que la incidencia de los incidentalomas aumente más debido a una mayor resolución de la TAC y a su uso indiscriminado ante la presencia de síntomas inespecíficos. Los autores recomiendan la utilización de métodos imaginológicos con posibilidades de definir el potencial de malignidad en los incidentalomas adrenales. La tomografía axial computarizada sin medio de contraste para el estudio del coeficiente de atenuación del incidentaloma adrenal es el método más aconsejado para diferenciar entre adenomas benignos o malignos, la hiperplasia, las lesiones quísticas, y los tumores malignos.

En todos los pacientes con incidentaloma adrenal está indicada la búsqueda del probable exceso hormonal. Los pacientes portadores de incidentalomas adrenales pobres en grasa se deben investigar para feocromocitomas, no importa si presentan o no hipertensión arterial. El hiperaldosteronismo primario debe sospecharse en pacientes hipertensos e hipocaliémicos. Se deben investigar los andrógenos adrenales en pacientes con signos de hiperandrogenismo. Se debe llevar a cabo la identificación de los incidentalomas adrenales, ya que se ha demostrado que estos tumores pueden tener relación con varios riesgos cardiovasculares. La cirugía está indicada para la mayoría de los incidentalomas adrenales hipersecretores y para aquellos sospechosos de malignidad. 


\section{Referencias}

1. Korobkin M, White EA, Kressel HY, et al. Computed tomography in the diagnoses of adrenal disease. AJRAm J Roentgenol. 1979; 132 (2): 231-238.

2. Young WF Jr. Management approaches to adrenal incidentalomas. A review from Rochester Minimesota. Endocrinal Metab Clin North Am. 2000; 29(1): 159-85

3. Kloos RT, Gross MD, Francis IR. Incidentally discovered adrenal masses. Endocr. 19955; 16(4): 460-84

4. Young WF.Jr. Clinical practice. The incintally discovered adrenal mass. N Engl J Med. 2007; 356 (6): 601-610.

5. Kawano M, Kodama T, Ito Y, et al. Adrenal incidentaloma-report of 14 operated cases and analysis of 4-year autopsy series of Japan. Nihon Geka Gakkai Zasshi. 1989; 90(12): 2031-2036.

6. Kannan S, Remer EM, Hamrahian AH. Evaluacion of patients with adrenal incidentalomas. Curr Opin Endorinol. Diabetes Obes. 2013; 20(3): 161-169.

7. Bovio S, Cataldi A, Reimondo G, et al. Prevalence of adrenal incidentaloma in a computarized tomograhy series. J Endocrinol F Invest, 2006; 29 (4): 298.

8. Davenport C, Liew A, Doherty B, et al. The prevalence of adrenal indicentaloma in routine clinical practice. Endocrine. 2011; 40(1): 80-83.

9. Mantero F, Terzolo M, Arnaldi G, et al. A survey on adrenal incidentaloma in Italy. Study group on adrenal tumors of The Italian Society of Endocrinology. J Clin Endocrinol Metab. 2000,85(2): 637-644.

10. Kim J, Bae KH, Choi YK, et al. Clinical Characteristics for 348 patients with adrenal incidentaloma. Endocrinol Metab (Seoul). 2012; 28(1): 20-25.

11. Kasperlik- Zaluska AA, Otto M, Cichocki A, et al. Incidentally discovered ad renal tumors: a lesson from observation of 1444 patients. Horm Metab Res. 2008: 40 (5): 338-341.

12. Bulow B, Janson S, Juhlin C, et al. Adrenal incidentaloma-follow-up results from a swedish prospective study . Eur J Endocrinol. 2006; 154(3): 419423

13. Terzolo M, Stigliano A, Chiodini I, et al. AME position statement on adrenal incidentaloma. Eur J Endocrinol. 2011; 164(6): 851-870.

14. Kim HY, Kimi SG, Lee KW, et al. Clinical study of adrenal incidentaloma in Korea. Korean J Intern Med. 2005; 20 (4): 303-309.

15. Barzon L, Fallo F, Sonino N, et al. Development of overt Cushing's síndrome in patients with adrenal incidentaloma. Eur J Endocrinol. 2002: 146(1): 61-

16. Angeli A, Osella G, Ali A, et al. Adrenal incidentaloma: An overview of data from The National Italian Study Group. Horm Res. 1997; 47 (4): 279-283.

17. Alguraan Z, Agcaoglu O, El- Hayek K, et al. Retroperitioneal masses mimicking adrenal tumors. Endocr Practice 2012; 18(3): 335-341.

18. Jaresch S, Kornely E, Kley HK, et al. Adrenal incidentaloma and patients with homozygous o heterozygous congenital adrenal hiperplasia. J clin Endocrinol Metab. 1992; 74(3): 685-689.

19. Barzon L, Sinino N,Fallo F, et al. Prevalence and natural history of adrenal incidentalomas. Eur J Endocrinol. 2003; 149(4): 273-285.

20. Musella M, Conzo G,Milone M, et al. Preoperative Work up in the assessment f adrenal incidentaloma: outcome from 282 consecutive laparascopic adrenalectomies. B MC Surg. 2013; 3:57-65.
21. Sturgeon C,Shen WT, Clark OH, et al. Risk assessment in 457 adrenal cortical carcinomas: how much tumor size predict the likelihood of malignancy? Am Coll Surg. 2006: 202 (3): 423-430.

22. McDermontt $\mathrm{S}, \mathrm{O}^{\prime}$ Connor $\mathrm{OJ}$, Cronin $\mathrm{OH}$, et al. Radiological evaluation of adrenal incidentalomas: currents methods and future prospects. Best Pract Res Clin Endocrinol Metab. 2012, 26(1): 21-33.

23. Hamrahian $\mathrm{AH}$, Loachescu AG, Remer EM, et al. Clinical utility of noncontrast computed tomography attenuation value (Hounsfield units) to: differentiate adrenal adenoma/hiperplasias from nonadenomas: Cleveland Clinic experience. J CLin Endocrinol Metab 2005; 90(2): 871-877.

24. Korobkin M, Brodeur FJ, Yutzy GG, et al. Diferentiation of adrenal adenomas from nonadenomas using CT attenuation values. AJR Am J Roentgenol 1996; 166(3): 531-536.

25. Lee MJ, Hahn PF, Papanicolaou N,et al. Benign and malignant adrenal masses: CT distinction with attenuation coefficients, size and abserver analysis. Radiology. 199; 179(2): 415-418.

26. Mayo- Smith WW, Boland Gw, Noto RB, et al. State-of-the art adrenal imaging. Radiographics. 2001;21(4): 995-1012.

27. Caoili EM, Korobkin M, Francis IR, et al. Adrena masses: characterization with combined unenhanced and delayed enhanced CT. Radiology. 2002;222(3): 629-633.

28. Kamiyama T, Fukukura Y, Yoneyama T, et al. Distinguishing adenomas from nonadenomas: Combined use of diagnstic parameters of unenhanced and short 5-minute dynamic enhaced CT protocol. Radiology. 2009; 250 (2): 471-481.

29. Korobkin M, Brodeur FJ, Francis IR, et al. Delayed enhanced CT for differen tiation of benign from malignant adrenal masses. Radiology. 1996;200(3): 737-742.

30. Timmers HJ, Chen CC, Carrasquillo JA, et al. Staging and funcional characterization of pheocromocytoma and paraganglioma by $18 \mathrm{~F}$-fluorodeoxyglucose (18F-FDG) positron emisión tomograhy . J Natl Cancer Inst. 2012 104(9): 700-.708.

31. Timmers HJ, Chen CC, Carrasquillo JA, et al. Comparison of $18 \mathrm{~F}$-fluoro-L DOPA, 18F-fluorodeoxyglucose, and 18F-fluorodopamine PET and 123 MIBG scintigraphy in the localization of preochromocytoma and paraganglioma. J Clin Endocrinol Metab. 2009; 94 (12) 4757-4767.

32. Ioachimescu GA, Remer ME, Hamrahian HA. Adrenal Incidentalomas. A dis ease of modern techhonology. Offering oportunities for improved patients care. Adrenal Cortical Neoplasia. 2015;44(2): 335-354

33. Herrera MF, Grant CS, Van Heerden JA, et al. Incidentally discovered adrenal tumor: an instutional perspective. Surgery. 1991; 110(6): 1014-1021.

34. Eldeiry LS, Garber JR. Adrenal Incidentalomas, 2003 to 2005: experience after publication of the National Institute of Health Consensus Statement. Endocr Pract. 2008;14(3): 279-284.

35. Morelli V, Reimondo G, Giordano R, et al. Long- term follow-up in adrenal incidentalomas: an Italian multicenter study. J Clin Endocrinol Metab. 2014;99(3): 827-834. 